International Journal of Pure and Applied Mathematics

Volume 93 No. 3 2014, 369-376

ISSN: 1311-8080 (printed version); ISSN: 1314-3395 (on-line version)

url: http://www.ijpam.eu

doi: http://dx.doi.org/10.12732/ijpam.v93i3.7

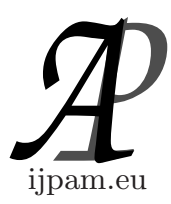

\title{
MAPPING ON GENERALIZED VAGUE SOFT EXPERT SET
}

\author{
Khaleed Alhazaymeh ${ }^{1}$, Nasruddin Hassan ${ }^{2} \S$ \\ ${ }^{1,2}$ School of Mathematical Sciences \\ Universiti Kebangsaan Malaysia \\ 43600 UKM, Bangi Selangor, MALAYSIA
}

\begin{abstract}
We introduce the mapping on generalized vague soft expert set and its operations are studied. The basic operations of mapping on generalized vague soft expert set theory are defined.
\end{abstract}

AMS Subject Classification: 03B52, 03E72

Key Words: fuzzy sot expert set, generalized fuzzy soft expert set, soft expert set, vague soft set

\section{Introduction}

The notion of mapping on soft classes are introduced by Kharal and Ahmad [1]. One of the most important new mathematical tools is soft set theory defined by Molodtsov [2]. This is extended to fuzzy soft sets [3], [4], [5], [6], [7] and then to vague soft sets [8], [9], [10], [11], [12] followed by interval valued vague soft sets [13], [14], [15]. Fuzzy sets were applied to genetic algorithms [16], [17] and into multi Q-fuzzy [18]. In this paper we introduce the notion of mapping on generalized vague soft expert classes and study the properties of generalized vague soft expert images and generalized vague soft expert inverse images of generalized vague soft expert sets. Finally, we give some examples of mapping on generalized vague soft expert classes.

Received: November 26, 2013

(C) 2014 Academic Publications, Ltd. url: www.acadpubl.eu

${ }^{\S}$ Correspondence author 


\section{Mapping on Generalized Vague Soft Expert Set}

In this paper, we introduce the notion of a mapping on generalized vague soft expert classes. Generalized vague soft expert classes are collections of generalized vague soft expert sets. We also define and study the properties of generalized vague soft expert images and generalized vague soft expert inverse images of generalized vague soft expert sets, and support them with example and theorems.

Definition 2.1. Let $U$ be a universe and $E$ be a set of parameters, $X$ a set of expert (agents) and $O=\{1=$ agree, $0=$ disagree $\}$ a set of opinions. Let $Z=E \times X \times O$. Then the collection of all generalized vague soft expert sets over $U$ with parameters from $Z$ is called a generalized vague soft expert class and is denoted as $\widetilde{(U, Z)}$.

Definition 2.2. Let $\widetilde{(U, Z)}$ and $\widetilde{(Y, \dot{Z})}$ be a generalized vague soft expert classes. Let $r: U \rightarrow Y$ and $s: Z \rightarrow Z$ be mappings. Then a mapping $f: \widetilde{(U, Z)} \rightarrow \widetilde{(Y, Z)}$ is defined as follows:

For a generalized vague soft expert set $\left(\tilde{F}_{\mu}, A\right) \in \widetilde{(U, Z)}, f\left(\tilde{F}_{\mu}, A\right) \in \widetilde{(Y, \dot{Z})}$ is a generalized vague soft expert set obtained as follows:

$f\left(\tilde{F}_{\mu}, A\right)(\beta)(y)=\left\{\begin{array}{cl}\left(\left\{\bigvee_{x \in r^{-1}\left(y_{i}\right), i=1,2 \ldots, n}\left(\bigvee_{\alpha} F(\alpha)\right)\right\}, \bigvee_{\alpha} \mu(\alpha)\right) & , \text { if } r^{-1}\left(y_{i}\right) \text { and } \\ (\phi, 0) & s^{-1}(\beta) \cap A \neq \emptyset\end{array}\right.$

for $\beta \in s(Z) \subseteq \dot{Z}, y_{i} \in Y$ and $\forall \alpha \in s^{-1}(\beta) \cap A, f\left(\tilde{F}_{\mu}, A\right)$ is called a generalized vague soft expert image of the expert vague soft set $\left.\left(\tilde{F}_{(} \mu\right), A\right)$.

Definition 2.3. Let $\widetilde{(U, Z)}$ and $\widetilde{(Y, \dot{Z})}$ be a generalized vague soft expert classes. Let $f^{-1}: \widetilde{(Y, \dot{Z})} \rightarrow \widetilde{(U, Z)}, r: U \rightarrow Y$ and $s: Z \rightarrow Z$ be mappings. For a generalized vague soft expert set $\left(\tilde{G}_{\delta}, B\right) \in \widetilde{(Y, \dot{Z})}$ where $B \subseteq Z^{\prime}, f^{-1}\left(\tilde{G}_{\delta}, B\right) \in$ $\widehat{(U, Z)}$ is a generalized vague soft expert set obtained as follows:

$$
f^{-1}\left(\tilde{G}_{\delta}, A\right)(\alpha)(u)=\left\{\begin{array}{cc}
\left(\left\{G(s(\alpha))\left(r\left(u_{j}\right)\right), \delta(s(\alpha))\right\}\right) & , \text { if } \alpha \in s^{-1}(B) \\
(\phi, 0) & \text {, otherwise }
\end{array}\right.
$$

for $\alpha \in s^{-1}(B) \subseteq Z$, and $u_{j} \in U, j=\{1,2, \ldots, m\} . f^{-1}\left(\tilde{G}_{\delta}, B\right)$ is called a generalized vague soft expert inverse image of the generalized vague soft expert set $\left(\tilde{G}_{\delta}, B\right)$. 
Example 2.1. Let $U=\left\{u_{1}, u_{2}, u_{3}\right\}, Y=\left\{y_{1}, y_{2}, y_{3}\right\}$ and let $A \subseteq Z=$ $\left\{\left(e_{1}, p, 1\right),\left(e_{2}, p, 0\right),\left(e_{3}, p, 1\right)\right\}$, and $A^{\prime} \subseteq Z^{\prime}=\left\{\left(e_{1}^{\prime}, p^{\prime}, 1\right),\left(e_{2}^{\prime}, p^{\prime}, 0\right),\left(e_{1}^{\prime}, q^{\prime}, 1\right)\right\}$.

Suppose that $\widetilde{(U, A)}$ and $\widetilde{\left(Y, A^{\prime}\right)}$ are generalized vague soft expert classes. Define $r: U \rightarrow Y$ and $s: A \rightarrow A^{\prime}$ as follows:

$r\left(u_{1}\right)=y_{1}, r\left(u_{2}\right)=y_{3}, r\left(u_{3}\right)=y_{1}$, $s\left(e_{1}, p, 1\right)=\left(e_{2}^{\prime}, p^{\prime}, 0\right), s\left(e_{2}, p, 0\right)=\left(e_{1}^{\prime}, p^{\prime}, 1\right), s\left(e_{3}, p, 1\right)=\left(e_{1}^{\prime}, p^{\prime}, 1\right)$.

Let $\left(\tilde{F}_{\mu}, A\right)$ and $\left(\tilde{G}_{\delta}, B\right)$ be two generalized vague soft expert sets over $U$ and $Y$ respectively such that

$$
\begin{aligned}
\left(\tilde{F}_{\mu}, A\right)=\{ & \left(\left(e_{1}, p, 1\right),\left\{\frac{u_{1}}{\langle 0.8,0.8\rangle}, \frac{u_{2}}{\langle 0.5,0.7\rangle}, \frac{u_{3}}{\langle 0.4,0.5\rangle}\right\}, 0.5\right), \\
& \left(\left(e_{3}, p, 1\right),\left\{\frac{u_{1}}{\langle 0.6,0.7\rangle}, \frac{u_{2}}{\langle 0.7,0.8\rangle}, \frac{u_{3}}{\langle 0.9,0.9\rangle}\right\}, 0.6\right), \\
& \left.\left(\left(e_{2}, p, 0\right),\left\{\frac{u_{1}}{\langle 0.7,0.8\rangle}, \frac{u_{2}}{\langle 0.1,0.6\rangle}, \frac{u_{3}}{\langle 0.2,0.5\rangle}\right\}, 0.7\right)\right\}, \\
\left(\tilde{G}_{\delta}, A^{\prime}\right)=\{ & \left(\left(e_{1}^{\prime}, p^{\prime}, 1\right),\left\{\frac{y_{1}}{\langle 0.7,0.7\rangle}, \frac{y_{2}}{\langle 0.4,0.6\rangle}, \frac{y_{3}}{\langle 0.3,0.4\rangle}\right\}, 0.4\right), \\
& \left(\left(e_{1}^{\prime}, q^{\prime}, 1\right),\left\{\frac{y_{1}}{\langle 0.5,0.6\rangle}, \frac{y_{2}}{\langle 0.6,0.7\rangle}, \frac{y_{3}}{\langle 0.8,0.8\rangle}\right\}, 0.5\right), \\
& \left.\left(\left(e_{2}^{\prime}, p^{\prime}, 0\right),\left\{\frac{y_{1}}{\langle 0.6,0.7\rangle}, \frac{y_{2}}{\langle 0,0.5\rangle}, \frac{y_{3}}{\langle 0.1,0.4\rangle}\right\}, 0.6\right)\right\} .
\end{aligned}
$$

Then we define the mapping from $f: \widetilde{(U, Z)} \rightarrow \widetilde{(Y, \dot{Z})}$ as follows:

For generalized vague soft expert set $\left(\tilde{F}_{\mu}, A\right)$ in $\overline{(X, Z)}, f\left(\left(\tilde{F}_{\mu}, A\right), K\right)$ is a generalized vague soft expert set in $\widetilde{(Y, \dot{E})}$ where $K=s(A)=\left\{\left(e_{1}^{\prime}, p^{\prime}, 1\right),\left(e_{2}^{\prime}, p^{\prime}, 0\right),\left(e_{1}^{\prime}, q^{\prime}, 1\right)\right\}$ and is obtained as follows:

$$
f\left(\tilde{F}_{\mu}, A\right)\left(e_{1}^{\prime}, p^{\prime}, 1\right)=\left(\left\{\underset{u \in r^{-1}\left(y_{i}\right), i=\{1,2,3\}}{\bigvee}\left(\bigvee_{\alpha} F(\alpha)\right)(x)\right\}, \bigvee_{\alpha} \mu(\alpha)\right)
$$

Now for $\left\{\bigvee_{u \in r^{-1}\left(y_{\mathrm{i}}\right), i=\{1,2,3\}}\left(\bigvee_{\alpha} F(\alpha)\right)(u)\right\}$ and for $i=1$, we have

$\underset{u \in\left\{u_{1}, u_{3}\right\}}{\bigvee}\left(\underset{\alpha \in\left\{\left(e_{2}, p, 0\right)\right\}}{\bigvee} F(\alpha)\right)(u)=\underset{u \in\left\{u_{1}, u_{3}\right\}}{\bigvee} F\left(e_{2}, p, 0\right)(u)$ 


$$
\begin{aligned}
& =\left\{\frac{u_{1}}{\langle 0.7,0.8\rangle}, \frac{u_{2}}{\langle 0.1,0.6\rangle}, \frac{u_{3}}{\langle 0.2,0.5\rangle}\right\}(u) \\
& =\langle 0.7,0.8\rangle \cup\langle 0.2,0.5\rangle=\langle 0.7,0.5\rangle
\end{aligned}
$$

For $i=2$,

$$
\begin{aligned}
& \bigvee_{u \in\left\{x_{2}\right\}}\left(\bigvee_{\alpha \in\left(e_{2}, p, 0\right)} F(\alpha)\right)(u)=0 \text { since } r^{-1}\left(y_{2}\right)=\phi \\
& \text { For } i=3 \text {, } \\
& \bigvee_{u \in\left\{u_{2}\right\}}\left(\bigvee_{\alpha \in\left\{e_{3}\right\}} F(\alpha)\right)(u)=\left(F\left(e_{1}\right)\right)\left(u_{2}\right) \\
& =\left(\left\{\frac{u_{1}}{\langle 0.7,0.8\rangle}, \frac{u_{2}}{\langle 0.1,0.6\rangle}, \frac{u_{3}}{\langle 0.2,0.5\rangle}\right\}\right)\left(u_{2}\right) \\
& =\langle 0.1,0.6\rangle \text {. }
\end{aligned}
$$

Now for $\bigvee \mu(\alpha)$, we have

$\bigvee_{e_{3}} \mu\left(e_{3}\right)=\mu\left(e_{3}\right)=0.7$.

Then

$$
f\left(\tilde{F}_{\mu}, A\right)\left(e_{2}^{\prime}, p^{\prime}, 0\right)=\left\{\left(e_{2}^{\prime}, p^{\prime}, 0\right),\left(\left\{\frac{y_{1}}{\langle 0.7,0.5\rangle}, \frac{y_{2}}{\langle 0,0\rangle}, \frac{y_{3}}{\langle 0.1,0.6\rangle}\right\}, 0.7\right)\right\}
$$

By similar calculations, consequently, we get

$$
\begin{aligned}
&\left(f\left(\tilde{F}_{\mu}, A\right), K\right)=\left\{\left(e_{1}^{\prime}, p^{\prime}, 1\right),\right.\left(\left\{\frac{y_{1}}{\langle 0.7,0.5\rangle}, \frac{y_{2}}{\langle 0,0\rangle}, \frac{y_{3}}{\langle 0.1,0.6\rangle}\right\}, 0.7\right) \\
&\left(e_{2}^{\prime}, p^{\prime}, 0\right),\left(\left\{\frac{y_{1}}{\langle 0.8,0.8\rangle}, \frac{y_{2}}{\langle 0,0\rangle}, \frac{y_{3}}{\langle 0.5,0.7\rangle}\right\}, 0.5\right), \\
&\left.\left(e_{1}^{\prime}, q^{\prime}, 1\right),\left(\left\{\frac{y_{1}}{\langle 0.9,0.7\rangle}, \frac{y_{2}}{\langle 0,0\rangle}, \frac{y_{3}}{\langle 0.7,0.8\rangle}\right\}, 0.6\right)\right\} .
\end{aligned}
$$

Next, for the generalized vague soft expert inverse images, we have the following:

For a generalized vague soft expert set $\left(\tilde{G}_{\delta}, A^{\prime}\right)$ in $(Y, Z)$,

$\left(f^{-1}\left(\tilde{G}_{\delta}, A^{\prime}\right), D\right)$ is a generalized vague soft expert set in $\widetilde{(U, Z)}$ where

$D=s^{-1}\left(A^{\prime}\right)=\left\{\left(e_{1}, p, 1\right),\left(e_{2}, p, 0\right),\left(e_{3}, p, 1\right)\right\}$ and obtained as follows:

$f^{-1}\left(\tilde{G}_{\delta}, B\right)\left(\left(e_{1}, p, 1\right)\right)\left(u_{1}\right)=G\left(e_{1}^{\prime}, p, 1\right)\left(r\left(u_{1}\right)\right)$

$$
\begin{aligned}
& =G\left(\left(e_{1}^{\prime}, p^{\prime}, 1\right)\right)\left(y_{1}\right) \\
& =\left\{\frac{y_{1}}{\langle 0.6,0.7\rangle}, \frac{y_{2}}{\langle 0,0.5\rangle}, \frac{y_{3}}{\langle 0.1,0.4\rangle}\right\}\left(y_{1},\right)=\langle 0.6,0.7\rangle .
\end{aligned}
$$

For $j=2$, we have $f^{-1}(G, B)\left(\left(e_{1}, p, 1\right)\right)\left(u_{2}\right)=G\left(\left(e_{1}^{\prime}, p^{\prime}, 1\right)\right)\left(y_{3}\right)$ 


$$
=\left\{\frac{y_{1}}{\langle 0.6,0.7\rangle}, \frac{y_{2}}{\langle 0,0.5\rangle}, \frac{y_{3}}{\langle 0.1,0.4\rangle}\right\}\left(y_{3}\right)=\langle 0.1,0.4\rangle .
$$

For $j=3$, we have $f^{-1}(G, B)\left(\left(e_{1}, p, 1\right)\right)\left(x_{3}\right)=G\left(e_{2}\right)\left(y_{1}\right)$

$$
=\left\{\frac{y_{1}}{\langle 0.6,0.7\rangle}, \frac{y_{2}}{\langle 0,0.5\rangle}, \frac{y_{3}}{\langle 0.1,0.4\rangle}\right\}\left(y_{1}\right)=\langle 0.6,0.7\rangle \text {. }
$$

Now for $\delta(s(\alpha))$, we have $s\left(e_{3}^{\prime}\right)=0.6$. Then

$f^{-1}\left(\tilde{G}_{\delta}, B\right)=\left(\left(e_{1}, p, 1\right),\left(\left\{\frac{y_{1}}{\langle 0.6,0.7\rangle}, \frac{y_{2}}{\langle 0.1,0.4\rangle}, \frac{y_{3}}{\langle 0.6,0.7\rangle}\right\}, 0.6\right)\right)$

By similar calculations, consequently, we get

$$
\begin{aligned}
&\left(f^{-1}\left(\tilde{G}_{\delta}, A\right), D\right)=\{\left(e_{1}, p, 1\right),\left(\left\{\frac{u_{1}}{\langle 0.5,0.6\rangle}, \frac{u_{2}}{\langle 0.8,0.8\rangle}, \frac{u_{3}}{\langle 0.5,0.6\rangle}\right\}, 0.5\right), \\
&\left(e_{2}, p, 0\right),\left(\left\{\frac{u_{1}}{\langle 0.6,0.7\rangle}, \frac{u_{2}}{\langle 0.1,0.4\rangle}, \frac{u_{3}}{\langle 0.6,0.7\rangle}\right\}, 0.6\right) \\
&\left.\left(e_{3}, p, 1\right),\left(\left\{\frac{u_{1}}{\langle 0.7,0.7\rangle}, \frac{u_{2}}{\langle 0.3,0.4\rangle}, \frac{u_{3}}{\langle 0.7,0.7\rangle}\right\}, 0.4\right)\right\} .
\end{aligned}
$$

Definition 2.4. Let $f: \widetilde{(U, X)} \rightarrow \widetilde{(Y, \dot{X})}$ be a mapping and $\left(\tilde{F}_{\mu}, A\right)$ and $\left(\tilde{G}_{\mu}, B\right)$ a generalized vague soft expert sets in $\widetilde{(X, E)}$. Then for $\beta \in \dot{X}$, the union and intersection of generalized vague soft expert images $\left(\tilde{F}_{\mu}, A\right)$ and $\left(\tilde{G}_{\delta}, B\right)$ are defined follows:

$$
\begin{aligned}
& \left(f\left(\tilde{F}_{\mu}, A\right) \widetilde{\bigvee} f\left(\tilde{G}_{\delta}, B\right)\right)(\beta)(y)=f\left(\tilde{F}_{\mu}, A\right)(\beta)(y) \widetilde{\bigvee} f\left(\tilde{G}_{\delta}, B\right)(\beta)(y) . \\
& \left(f\left(\tilde{F}_{\delta}, A\right) \widetilde{\bigwedge} f\left(\tilde{G}_{\delta}, B\right)\right)(\beta)(y)=f\left(\tilde{F}_{\mu}, A\right)(\beta)(y) \widetilde{\bigwedge} f\left(\tilde{G}_{\delta}, B\right)(\beta)(y) .
\end{aligned}
$$

Definition 2.5. Let $f: \widetilde{(U, X)} \rightarrow \widetilde{(Y, \dot{X})}$ be a mapping and $\left(\tilde{F}_{\mu}, A\right)$ and $\left(\tilde{G}_{\delta}, B\right)$ a generalized vague soft expert sets in $\widetilde{(X, E)}$. Then for $\beta \in \dot{X}$, the union and intersection of generalized vague soft expert images $\left(\tilde{F}_{\mu}, A\right)$ and $\left(\tilde{G}_{\delta}, B\right)$ are defined follows:

$$
\begin{aligned}
& \left(f^{-1}\left(\tilde{F}_{\mu}, A\right) \widetilde{\bigvee} f^{-1}\left(\tilde{G}_{\delta}, B\right)\right)(\alpha)(u)=f^{-1}\left(\tilde{F}_{\mu}, A\right)(\alpha)(u) \widetilde{\bigvee} f^{-1}\left(\tilde{G}_{\delta}, B\right)(\alpha)(u) . \\
& \left(f^{-1}\left(\tilde{F}_{\mu}, A\right) \widetilde{\bigwedge} f^{-1}\left(\tilde{G}_{\delta}, B\right)\right)(\alpha)(u)=f^{-1}\left(\tilde{F}_{\mu}, A\right)(\alpha)(u) \widetilde{\bigwedge} f^{-1}\left(\tilde{G}_{\delta}, B\right)(\alpha)(u) .
\end{aligned}
$$


Proposition 2.1. Let $f: \widetilde{(U, X)} \rightarrow \widehat{(Y, X)}$ be a mapping. Then for generalized vague soft expert sets $\left(\tilde{F}_{\mu}, A\right)$ and $\left(\tilde{G}_{\delta}, B\right)$ in the generalized vague soft expert class $\widetilde{(U, X)}$,

1. $f(\phi)=\phi$.

2. $f(X) \subseteq Y$.

3. $f\left(\left(\tilde{F}_{\mu}, A\right) \tilde{\bigvee}\left(\tilde{G}_{\delta}, B\right)\right)=f\left(\tilde{F}_{\mu}, A\right) \tilde{\vee} f\left(\tilde{G}_{\delta}, B\right)$.

4. $f\left(\left(\tilde{F}_{\mu}, A\right) \tilde{\wedge} f\left(\tilde{G}_{\delta}, B\right)\right) \subseteq f\left(\tilde{F}_{\mu}, A\right) \tilde{\wedge} f\left(\tilde{G}_{\delta}, B\right)$.

5. If $\left(\tilde{F}_{\mu}, A\right) \subseteq\left(\tilde{G}_{\delta}, B\right)$, thenf $\left(\tilde{F}_{\mu}, A\right) \subseteq f\left(\tilde{G}_{\delta}, B\right)$.

Proof. The proof is straightforward.

Proposition 2.2. Let $f: \widetilde{(U, X)} \rightarrow \widetilde{(Y, \dot{X})}$ be a mapping. Then for generalized vague soft expert sets $\left(\tilde{F}_{\mu}, A\right),\left(\tilde{G}_{\delta}, B\right)$ in the generalized vague soft expert class $(U, \dot{X})$, we have:

1. $f^{-1}(\emptyset)=\phi$.

2. $f^{-1}(Y)=X$.

3. $f^{-1}\left(\left(\tilde{f}_{\mu}, A\right) \tilde{\bigvee}\left(\tilde{G}_{\delta}, B\right)\right)=f^{-1}\left(\tilde{F}_{\mu}, A\right) \tilde{\bigvee} f^{-1}\left(\tilde{G}_{\delta}, B\right)$.

4. $f^{-1}\left(\left(\tilde{f}_{\mu}, A\right) \tilde{\wedge}\left(\tilde{G}_{\delta}, B\right)\right)=f^{-1}\left(\tilde{F}_{\mu}, A\right) \tilde{\wedge} f\left(\tilde{G}_{\delta}, B\right)$.

5. If $\left(\tilde{f}_{\mu}, A\right) \subseteq\left(\tilde{G}_{\delta}, B\right)$, thenf $f^{-1}\left(\tilde{f}_{\mu}, A\right) \subseteq f^{-1}\left(\tilde{G}_{\delta}, B\right)$.

Proof. The proof is straightforward.

\section{Conclusion}

In this paper, we studied a mapping on generalized vague soft expert classes and its properties. We give some illustrative examples of mapping on generalized vague soft expert set. 


\section{Acknowledgments}

We are indebted to Universiti Kebangsaan Malaysia for funding this research under the grant BKBP-FST-K005560.

\section{References}

[1] A. Kharal and B. Ahmad, Mappings on soft classes, New Mathematics and Natural Computation, 07 (3) (2011), 471-481.

[2] D. Molodtsov, Soft set theory-first result, Computers and Mathematics with Applications, 37 (1999), 19-31.

[3] S. Alkhazaleh. A. R. Salleh and N. Hassan, Soft multisets theory, Applied Mathematical Sciences, 5 (72) (2011), 3561-3573.

[4] S. Alkhazaleh. A. R. Salleh and N. Hassan, Possibility fuzzy soft set, Advances in Decision Sciences, Article ID 479756, 18 pages, (2011) doi:10.1155/2011/479756.

[5] S. Alkhazaleh. A. R. Salleh and N. Hassan, Fuzzy parameterized intervalvalued fuzzy soft set, Applied Mathematical Sciences, 5 (67) (2011), 33353346 .

[6] A. R. Salleh, S. Alkhazaleh, N. Hassan and A.G. Ahmad, Multiparameterized soft set, Journal of Mathematics and Statistics, 8 (1) (2012), 92-97.

[7] K. Alhazaymeh, S.A. Halim, A. R. Salleh, and N. Hassan, Soft intuitionistic fuzzy sets, Applied Mathematical Sciences, 6 (54) (2012), 2669-2680.

[8] K. Alhazaymeh and N. Hassan, Generalized vague soft set and its applications, International Journal of Pure and Applied Mathematics, 77 (3) (2012), 391-401.

[9] K. Alhazaymeh and N. Hassan, Possibility vague soft set and its application in decision making, International Journal of Pure and Applied Mathematics, 77 (4) (2012), 549-563.

[10] N. Hassan and K. Alhazaymeh, Vague soft expert set theory, AIP Conference Proceedings, 1522 (2013), 953-958 ; doi: 10.1063/1.4801233.

[11] K.Alhazaymeh and N.Hassan, Generalized vague soft expert set,International Journal of Pure and Applied Mathematics, (in press). 
[12] K. Alhazaymeh and N. Hassan, Application of generalized vague soft expert set in decision making, International Journal of Pure and Applied Mathematics, (in press).

[13] K. Alhazaymeh and N. Hassan, Interval-valued vague soft sets and its application, Advances in Fuzzy Systems, Article ID 208489, 7 pages, (2011), doi:10.1155/2012/208489.

[14] K.Alhazaymeh and N.Hassan, Possibility interval-valued vague soft set, Applied Mathematical Sciences, 7 (140) (2013), 6989-6994.

[15] K.Alhazaymeh and N.Hassan, Generalized interval-valued vague soft set, Applied Mathematical Sciences, 7 (140) (2013), 6983-6988.

[16] M. Varnamkhasti and N. Hassan, A hybrid of adaptive neuro-fuzzy inference system and genetic algorithm, Journal of Intelligent and Fuzzy Systems, 25 (3) (2013), 793-796.

[17] M. Varnamkhasti and N. Hassan, Neurogenetic algorithm for solving combinatorial engineering problems, Journal of Applied Mathematics, Article ID 253714, 12 pages (2012), doi:10.1155/2012/253714.

[18] F. Adam and N. Hassan, Multi Q-fuzzy parameterized soft set and its application, Journal of Intelligent and Fuzzy System, (in press). 